\title{
Inherited and de novo mutations in sporadic cases of DYT1-dystonia
}

\author{
Lena Elisabeth Hjermind ${ }^{1,2}$, Lene Moria Werdelin ${ }^{2}$ and Sven Asger Sørensen*,1 \\ ${ }^{1}$ Department of Medical Genetics, The Panum Institute, University of Copenhagen, Copenhagen, Denmark; \\ ${ }^{2}$ Department of Neurology, Bispebjerg Hospital, Copenhagen, Denmark
}

A study of Danish probands with primary torsion dystonia is presented. The probands were examined clinically and biochemically to exclude secondary dystonia. Mutation analyses for the GAG-deletion in the DYT1 gene were performed on 107 probands; and the mutation was detected in three. All three probands had the classical phenotype of DYT1-dystonia, but only one had a family history of dystonia. The other two probands had, obviously, sporadic DYT1-dystonia, one of which was caused by a de novo mutation, while the other one had a parent being an asymptomatic carrier. De novo mutations in the DYT1 gene are seldomly reported although independent founder mutations are known to have occurred. The frequency of DYT1dystonia was low in our study even though several probands had early onset generalised dystonia. None of the probands in our study with other types of dystonia had the GAG-deletion as reported in other studies. The difficulties in genetic counselling concerning the heterogeneity of dystonia examplified by DYT1dystonia are outlined.

European Journal of Human Genetics (2002) 10, 213 -216. DOI: 10.1038/sj/ejhg/5200782

Keywords: primary torsion dystonia; DYT1 gene; de novo mutation; hereditary; non-penetrance

\section{Introduction}

Dystonia is a heterogenous, neurological disease characterised by involuntary, sustained muscle contractions, frequently causing twisting and repetitive movements or abnormal postures. ${ }^{1}$

It is believed that about $70 \%$ of all patients with dystonia have idiopathic or primary dystonia rather than symptomatic or secondary. ${ }^{1}$ Investigations have indicated that $25 \%$ of all cases of focal dystonia are hereditary, ${ }^{2}$ and that $85 \%$ of all cases of generalised, multifocal, and segmental dystonia are hereditary. ${ }^{3}$

There are at least 11 different gene loci for autosomal, dominant inherited dystonia, one for autosomal, recessive inherited dystonia and one for X-linked, recessive inherited dystonia, but only four genes have been cloned:

*Correspondence: Sven Asger Sørensen, Department of Medical Genetics, The Panum Institute, Blegdamsvej 3, DK-2200 Copenhagen N, Denmark. Tel: +45 35327830; Fax: +45 35327845; E-mail: sas@imbg.ku.dk Received 15 November 2001; revised 3 January 2002; accepted 4 January 2002
DYT1, DYT5 (two genes, at 14q22.1 coding for the autosomal dominant dopa responsive dystonia, and at 11 p15.5 coding for autosomal recessive) and DYT11. ${ }^{4-6}$ (at 7q21 coding for autosomal dominant Myoclonus-Dystonia Syndrome).

Clinically, DYT1-dystonia is often described as early limb-onset generalised dystonia, ${ }^{7-9}$ but further investigations have shown that both onset of symptoms and expression of the disease in DYT1-positive patients can vary with onset up to 44 years and disease duration ranging up to 30 years without spread of symptoms. ${ }^{10,11}$ DYT1-dystonia is by clinical and later also molecular genetic studies shown to be inherited as an autosomal, dominant trait with 30-40\% penetrance and localised on chromosome 9q34. ${ }^{12}$ The gene encodes an ATP-binding protein, TorsinA. ${ }^{8}$ This protein is a member of the AAA family of ATPases, often performing chaperone-like functions that assist in the assembly, operation or disassembly of protein complexes. It has been suggested that TorsinA may function as a motor protein in the transport of dopamine-containing membranous vesicles. ${ }^{13}$ 


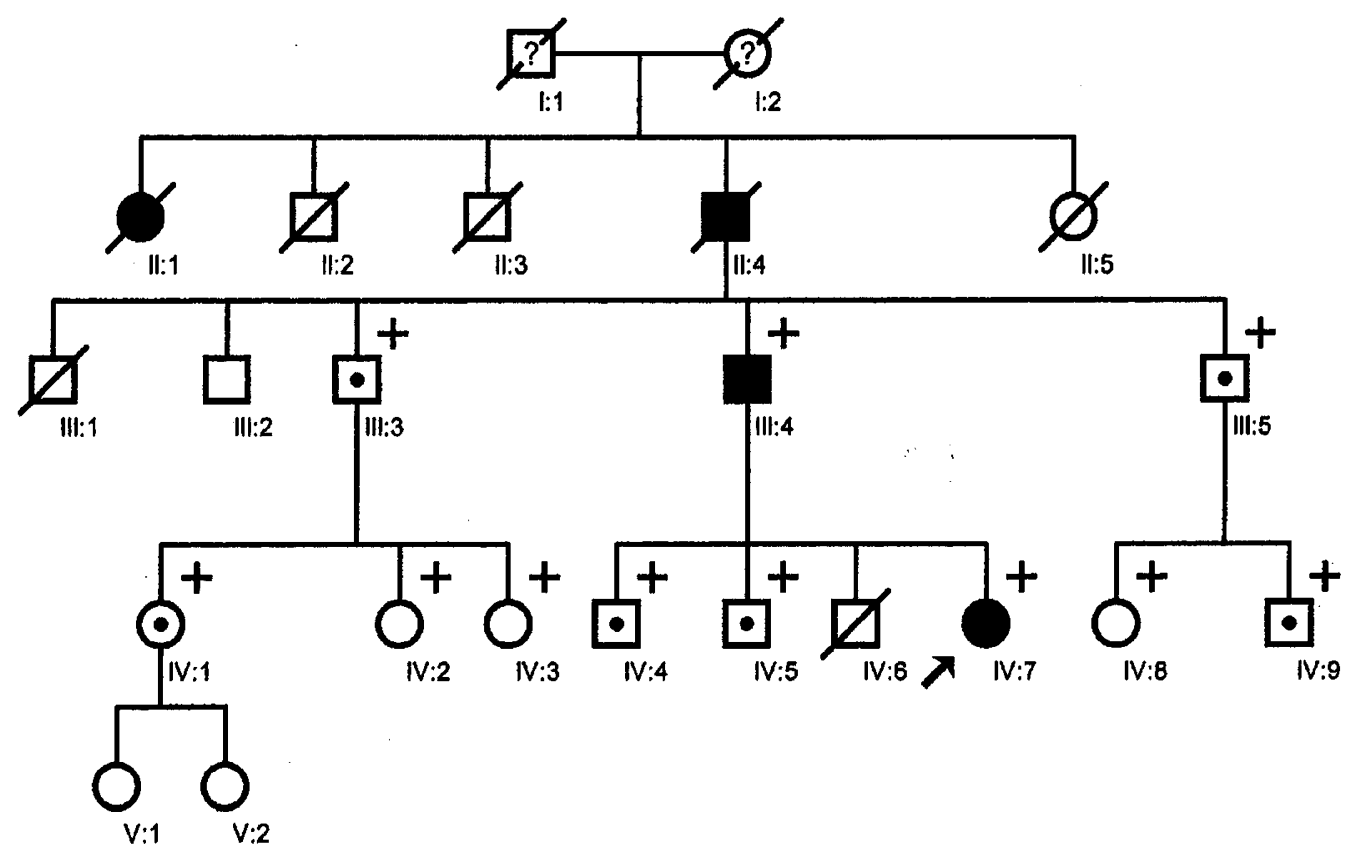

1 Affected

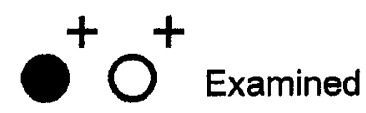

\section{○ Non-affected carrier}

Figure 1 Pedigree of family with DYT1 dystonia.

The mutation in the DYT1 gene is a GAG-deletion at the position 946, and the different haplotypes among families with the GAG-deletion suggest that at least six independent founder mutations have occurred. ${ }^{10}$ However, another mutation in the DYT1 gene has recently been reported. This novel 18 base pair deletion is the first additional mutation and seems to be associated with a distinct type of early onset dystonia. $^{14}$

A de novo GAG-deletion in the DYT1 gene has been reported in two patients of Swiss-Mennonite and non-Jewish Russian origin, respectively; ${ }^{15}$ and suggested in a patient of German origin. ${ }^{16}$

The purpose of the present study is to report on the frequency of DYT1 in a Danish population of probands with primary torsion dystonia. Further to report on the sporadic cases caused by reduced penetrance or de novo mutations with respect to genetic counselling.

\section{Materials and methods}

Clinical and genetical examinations were done in a study of 112 unrelated Danish probands with primary torsion dystonia. Primary dystonia was defined as dystonia without any known causes and without any associated neurological symptoms. Electromyography was performed in all probands except four and showed the characteristic dystonic changes; CT-scan of cerebrum, MRI of cerebrum and both a CT-scan and a MRI in 30, 28 and four, respectively were normal.

In the beginning of the study probands were selected from hospital files at hospitals in Copenhagen and Aarhus covering patients from most of Denmark - and later also probands having heard about the study asked for admission themselves. All probands were seen regularly at departments of neurology. The probands were examined from January 1999 to September 2001. 
Five probands were excluded, because of doubt whether their disease was primary or secondary dystonia. The probands were 27 men and 80 women; mean age 50.5 years (range: $21-81$ ) and mean age at onset 31.8 years (range: $0-$ 80). The reason for the excess of female probands was that more women applied for admission to the study. Thirty-seven were known familial cases. The relatives of 13 probands were known familial cases, while 24 probands reported on dystonic symptoms in relatives who were deceased or for other reasons not available.

Blood samples were collected for biochemical analyses and extraction of genomic DNA. Biochemically a standard set of tests analysing red and white blood cell count, liver- and kidney function, antinuclear antibodies, copper in serum and ceruloplasmin were performed. Mutation analyses for the GAG-deletion in the DYT1 gene on chromosome $9 \mathrm{q} 34$ were performed as described earlier. $^{8}$

We analysed for the mutation in both familial and sporadic cases, because of the low penetrance of the DYT1 allele.

Paternity testing was done by use of the D1S80 VNTRsystem. ${ }^{17}$

\section{Results}

The clinical examinations showed that 22 probands had generalised dystonia, two had hemidystonia, five had multifocal dystonia, 15 had segmental dystonia, and 63 had focal dystonia. Among the 22 probands with generalised dystonia 20 had early limb-onset generalized dystonia with 13 years (range: 40 days -40 years) as the mean age of onset. The first symptoms appeared in the lower extremities in 10 probands, in the upper extremities in seven probands and unknown in the remaining three.

Among the 107 probands investigated we only detected the DYT1-mutation in three $(2.8 \%)$ in whom the phenotype was typical. These three correspond to $15 \%$ of the 20 probands with typical phenotype. Concerning family status the DYT1mutation was only detected in one proband with familial dystonia out of 37 (2.7\%).

The proband with the DYT1-mutation and a positive family history had relatives in two earlier generations with dystonia. The mutation was also encountered in her father, who is the only relative alive with clinical symptoms of dystonia and in six asymptomatic adult relatives (Figure 1 ).

In order to determine whether the two sporadic cases were de novo mutations or due to the low penetrance of the DYT1 gene, mutation analyses were performed on the parents of the two probands with no family history of dystonia. In one case the DYT1-mutation was encountered in the mother of the proband, while none of the parents of the other case had a GAG-deletion. Paternity testing revealed that non-paternity was unlikely.

\section{Discussion}

The preliminary investigations of idiopathic torsion dystonia in Denmark indicate a low frequency of DYT1 dystonia as the mutation was only encountered in three among 107 unrelated probands $(2.8 \%)$, or $15 \%$ among 20 with early onset, generalised dystonia. Thirty-seven probands had a positive family history; four of them with the typical DYT1 phenotype, but only one of the probands with the DYT1-mutation had a positive family history.

This is in contrast to the higher frequency of the GAGdeletion found in patients with early onset, generalised dystonia and/or a positive family history in other studies. ${ }^{8,10,18-20}$

We only found the GAG-deletion in probands with early limb-onset, generalised dystonia; but the mutation was not found in several probands with similar symptoms. Phenotypic variability has been reported; eg writer's cramp has been reported as the only manifestation of probands carrying the mutation in the DYT1gene. ${ }^{10,15,21}$

In our study one out of two sporadic cases had a de novo mutation in the DYT1 gene, while the other one had inherited the mutation from the mother, who had no signs of dystonia because of the low penetrance.

Founder mutations in different populations have been reported, ${ }^{10}$ but de novo mutations in the DYT1 gene have been reported only once in two patients from different populations $^{15}$ and suggested in another patient from a third population, where DNA from the parents was not available. ${ }^{16}$ Solitary cases of DYT1-dystonia have been reported in studies where molecular analyses of the DNA of the parents were not done. ${ }^{10,11}$ Apparently sporadic cases because of the low penetrance have also been reported earlier. ${ }^{20}$

The difficulties in genetic counselling concerning dystonia is partly due to the low penetrance of many of the hereditary forms of dystonia and partly due to the variable phenotype within the same type of dystonia and, as shown in our study, the occurrence of de novo mutations.

An exact genetic diagnosis is crucial for genetic counselling and cannot be obtained by clinical examination alone because of the phenotypic variability, the low penetrance and the occurrence of de novo mutations. It has been argued not to do diagnostic DYT1 testing on patients without affected relatives with onset at age 26 years or later. $^{22}$ The DYT1-mutation, however, has been reported in solitary as well as phenotypically non-typical cases of dystonia. ${ }^{10,15,21}$ The question can therefore be raised whether all patients with primary torsion dystonia, irrespective of symptoms - sporadic as well as familial cases - by routine should be offered a mutation analysis for DYT1 and/or any of the other gene loci for inherited dystonia. 
13 Neuwald AF, Aravind L, Spouge JL, Koonin EV: AAA+: a class of chaperone-like ATPases associated with the assembly, operation, and disassembly of protein complexes. Genome Res 1999; 9 : $27-43$.

14 Leung JC, Klein C, Friedman J et al: Novel mutation in the TOR1A (DYT1) gene in atypical early onset dystonia and polymorphisms in dystonia and early onset parkinsonism. Neurogenet 2001; 3: $133-143$.

15 Klein C, Brin MF, de Leon D et al: De novo mutation (GAG deletion) in the DYT1 gene in two non-Jewish patients with early-onset dystonia. Hum Mol Genet 1998; 7: 1133-1136.

16 Leube B, Kessler KR, Ferbert A et al: Phenotypic variability of the DYT1 mutation in German dystonia patients. Acta Neurol Scand 1999; 99: $248-251$.

17 Thymann M, Nellemann LJ, Masumba G, Irgens-Møller L, Morling N: Analysis of the locus D1S80 by amplified fragment length polymorphisms technique (AMP-FLP). Frequency distribution in Danes. Intra and inter laboratory reproducibility of the technique. Forensic Sci Int 1993; 60: 47-56.

18 Bressman SB, de Leon MS, Kramer PL et al: Dystonia in Ashkenazi Jews: clinical characterization of a founder mutation. Ann Neurol 1994; 36: 771 - 777.

19 Klein C, Friedman J, Bressman S et al: Genetic testing for earlyonset torsion dystonia (DYT1): introduction of a simple screening method, experiences from testing of a large patient cohort, and ethical aspects. Genet Test 1999; 3: 323-328.

20 Brassat D, Camuzat A, Vidialhet M et al: Frequency of the DYT1 mutation in primary torsion dystonia without family history. Arch Neurol 2000; 57: $333-335$.

21 Gasser T, Windgassen K, Bereznai B, Kabus C, Ludolph AC. Phenotypic expression of the DYT1 mutation: a family with writer's cramp of juvenile onset. Ann Neurol 1998; 44: 126-128.

22 Bressman SB, Sabatti C, Raymond D et al: The DYT1 phenotype and guidelines for diagnostic testing. Neurology 2000; 54: $1746-$ 1752 . 